\title{
PEKING, TAIPEI UND DIE UNO: ANALYSE DES NEUEN ABSTIMMUNGSERGEBNISSES ÜBER DIE FRAGE DER CHINESISCHEN REPRÄSENTATION UND DIE REAKTION TAIPEIS
}

\author{
Von Y. H. NiEH
}

Die Regierung in Taipei mißt der Verteidigung der eigenen Position in der UNO jedes Jahr fast denselben Stellenwert bei wie bei der politischen Richtlinie der „Rückeroberung des (chinesischen) Festlands“. Deshalb spricht man nicht selten von „einem diplomatischen Kampf“, einem Kampf mit Peking um die legitime Repräsentation des chinesischen Volkes auf der internationalen politischen Bühne.

Als die Kommunistische Partei Chinas 1949 das gesamte Gebiet Chinas bis auf die Provinz Taiwan und einige andere Inseln an der Küste des chinesischen Festlands eroberte und die neue Volksrepublik in Peking gründete, konnte sie in der Diplomatie jedoch nur einen bescheidenen Erfolg erzielen. Lediglich 10 Ostblockstaaten haben damals die Regierung in Peking sofort anerkannt. Im folgenden Jahr bemühten sich die Sowjetunion und Indien im Sicherheitsrat und in der Vollversammlung der UNO vergeblich darum, die Aufnahme Pekings in und gleichzeitig den Ausschluß Taipeis aus der Weltorganisation zu erreichen ${ }^{1}$.

Die Vollversammlung der UNO hat sich in einer Resolution vom 14. Dezember 1950 das Recht auf Entscheidung vorbehalten, wenn es über die Frage der Repräsentation eines Mitgliedstaats - im Unterschied zur Aufnahme eines neuen Mitgliedstaats - zur Kontroverse in der UNO kommen sollte². Seitdem wird über die Frage der rechtmäßigen Vertretung Chinas jedes Jahr in der Vollversammlung abgestimmt; eine Ausnahme gab es nur 1964, als die UNO wegen des Finanzdisputs über den Unterhalt der UNO-Truppen zwischen den Vereinigten Staaten und der Sowjetunion nicht zur Chinafrage kommen konnte.

Offensichtlich war der Korea-Krieg daran schuld, daß die Anträge der Sowjetunion über die Aufnahme Pekings und den Ausschluß Taipeis in der ersten Hälfte der fünfziger Jahre nur wenige Stimmen gewinnen konnten. Doch die außenpolitische Aktivität Pekings in der Dritten Welt nach dem Korea-Krieg, besonders aber nach der Bandung-Konferenz von 1955, brachte im Verlauf der letzten Hälfte des Jahrzehnts bereits eine ständige Stimmenzunahme in der UNO, obwohl die USA alle auf Ersetzung Taipeis durch Peking abzielenden Versuche der Sowjetunion und Indiens bis 1960 mit einem Verfahrensantrag von Jahr zu Jahr blockieren und aufschieben konnten.

Angesichts des Zustroms neuer afrikanischer Mitgliedstaaten zur UNO Anfang der sechziger Jahre rückten die Amerikaner von ihrer Moratoriumspolitik ab und änderten 1961 zum ersten Mal ihre Taktik zur Chinafrage. Seitdem wollen sie nicht mehr verhindern, daß der Antrag auf Aufnahme Pekings auf die Tagesordnung der Vollversammlung gesetzt wird, sondern zielen darauf ab, den Streit um

\footnotetext{
1 Vgl. dazu China Handbook 1951, Taipei 1951, S. 106 ff; Winberg Chai: China and the United Nations: Problems of Representation and Alternatives, in: Asian Survey, 1970, S. $399 \mathrm{f}$.

2 China Handbook 1951, a. a. O., S. 108 f. Zur Aufnahme eines neuen Mitgliedstaates bedarf es gemäß der UNO-Satzung (Art. 4 Abs. 1 und Art. 18 Abs. 2) eines Beschlusses des Sicherheitsrates - der vetoabhängig ist - und eines daraufhin ergehenden Beschlusses der Vollversammlung mit Zweidrittelmehrheit.
} 
die chinesische Repräsentation durch einen Prozedurantrag zu einer "wichtigen Frage" zu erheben, wodurch eine Zweidrittelmehrheit gemäß Art. 18 der UNOSatzung erforderlich wird.

Aufgrund der von Frankreich ausgelösten Anerkennungswelle (1964-65) konnte Peking bei der Abstimmung über die chinesische Repräsentation in der 20. Sitzungsperiode der Vollversammlung erneute Erfolge verbuchen. Die Sympathiestimmen für Peking haben 1965 ein Gleichgewicht mit den Gegenstimmen errungen (47:47). Doch zwischen 1966 und 1968 hat der Einfluß der Großen Proletarischen Kulturrevolution die Stimmenzahl für Peking wieder rückläufig gemacht. Erst 1969 zeigte die Pro-Peking-Kurve einen erneuten Anstieg. 1970 durchbrach sie - mit einem Ergebnis von 51 Ja- gegen 49 Nein-Stimmen für Peking - zum ersten Mal seit 20 Jahren die Grenze der einfachen Mehrheit, erreichte allerdings immer noch nicht die für die Aufnahme Pekings notwendige Zweidrittelmehrheit. (Der amerikanische Antrag, die Aufnahme Pekings zu einer „wichtigen Frage“ zu erklären, war ja schon vorher mit 66 zu 52 Stimmen gutgeheißen worden.)

Entwicklung des Stimmenverhältnisses 1951—1970

\begin{tabular}{|c|c|c|c|c|c|c|c|}
\hline \multirow[b]{2}{*}{ Jahr } & \multicolumn{3}{|c|}{ Aufnahme Pekings } & \multicolumn{3}{|c|}{ Wichtige Frage } & \multirow{2}{*}{$\begin{array}{l}\text { Zahl der Mitglied- } \\
\text { staaten insgesamt }\end{array}$} \\
\hline & $\mathrm{Ja}$ & Nein & neutral* & $\mathrm{Ja}$ & Nein & neutral* & \\
\hline 1951 & 11 & 37 & 4 & & & & 60 \\
\hline 1952 & 9 & 42 & 11 & & & & 60 \\
\hline 1953 & 10 & 44 & 6 & & & & 60 \\
\hline 1954 & 11 & 43 & 6 & & & & 60 \\
\hline 1955 & 12 & 42 & 6 & & & & 60 \\
\hline 1956 & 24 & 47 & 8 & & & & 79 \\
\hline 1957 & 27 & 48 & 7 & & & & 82 \\
\hline 1958 & 28 & 44 & 9 & & & & 81 \\
\hline 1959 & 29 & 44 & 9 & & & & 82 \\
\hline 1960 & 34 & 42 & 22 & & & & 98 \\
\hline 1961 & 36 & 48 & 20 & 61 & 34 & 9 & 104 \\
\hline 1962 & 42 & 56 & 12 & & in Ant & ag) & 110 \\
\hline 1963 & 41 & 57 & 13 & & in Ant & ag) & 111 \\
\hline 1964 & \multicolumn{7}{|c|}{ (keine Abstimmung abgehalten) } \\
\hline 1965 & 47 & 47 & 23 & 56 & 49 & 12 & 117 \\
\hline 1966 & 46 & 57 & 18 & 66 & 48 & 7 & 121 \\
\hline 1967 & 45 & 58 & 19 & 69 & 48 & 5 & 122 \\
\hline 1968 & 44 & 58 & 24 & 73 & 47 & 6 & 126 \\
\hline 1969 & 48 & 56 & 22 & 71 & 48 & 7 & 126 \\
\hline 1970 & 51 & 49 & 27 & 66 & 52 & 9 & 127 \\
\hline
\end{tabular}

* Stimmenthaltung, Nichtbeteiligung und Nichtanwesenheit 
Obwohl Peking auf seinem „Langen Marsch“ nach New York noch einen "großen Sprung" machen muß, bis es die von Washington errichtete Schwelle der Zweidrittelmehrheit überschreiten kann, hat die neue Entwicklung in der UNO in Taipei tiefe Beunruhigung ausgelöst. Bereits 1969 hatte Taiwan den Sieg von 56 gegen 48 Stimmen gegen die Aufnahme Pekings und von 71 gegen 48 Stimmen für die "wichtige Frage“ (Anwendung des Art. 18 der UNO-Satzung über die Zweidrittelmehrheit) nicht ohne Sorge zur Kenntnis genommen. Die Presse auf der Insel machte seinerzeit die "internationale Beschwichtigungspolitik“ und besonders die „unentschlossene Führung Amerikas in der freien Welt" für die Tendenzen der Stimmeneinbuße verantwortlich ${ }^{3}$. Diesmal nimmt man den letzten Erfolg noch erbitterter hin. Es wird darauf hingewiesen, daß die internationale Gesellschaft unter dem Einfluß eines „Tiefdrucks“ oder einer "Gegenströmung“ steht".

In einer Analyse der Chung-yang Jih-pao, des Organs der Regierungspartei, zählt der Journalist Yü Szu-chou die folgenden Gründe auf, die die letzte Abstimmung über die chinesische Vertretungsfrage in der UNO beeinflußt haben sollen. Vor allen Dingen meint er, daß die internationale Konferenz der blockfreien Länder in Lusaka, der Hauptstadt von Sambia, noch vor der Einberufung der UNO-Vollversammlung erklärt habe, Peking bei der Aufnahme in die Weltorganisation unterstützen zu wollen. Dies habe sich merklich auf die Haltung der afrikanischen Staaten ausgewirkt. Zweitens feierte die UNO 1970 den 25. Jahrestag ihrer Gründung; viele Staatsoberhäupter und Regierungsführer hätten in ihren Jubiläumsreden zu diesem Anlaß das „Universalitätsprinzip der UNO“ betont und die pekingfreundlichen Staaten hätten dies mit der Aufnahme Pekings verknüpft. Als dritter Grund für die Stimmenzunahme Pekings in der UNO gilt nach Yü die neue Anerkennung Pekings durch Kanada und Italien, die auch den Standpunkt von Ländern wie Irland, Luxemburg und Österreich zur Chinafrage in der UNO weiter beeinflußt habe. Yü meint zum vierten Punkt, daß Frankreich unter den französischsprachigen afrikanischen Ländern für Peking geworben habe. Auch Pekings neue "lächelnde Diplomatie“ und die Wiederbesetzung der zahlreichen durch die Kulturrevolution vakant gewordenen Botschafterposten im Ausland - der fünfte Punkt von Yü - habe in der UNO Sympathien geschaffen. Da sich die Beziehungen zwischen Peking und Moskau auf dem Wege der Normalisierung befinden, plädierte auch der sowjetische Vertreter in der UNO nach einem Schweigen von zwei Jahren diesmal wieder für Peking; dies ist der sechste Punkt in Yüs Analyse. Als siebte Ursache des neuen Stimmengewinns für den pekingfreundlichen Antrag in der Vollversammlung führt Yü die Machtübernahme linksgerichteter Parteien in vielen Ländern, wie Chile, Bolivien und Osterreich, an. Zum letzten behauptet der nationalchinesische Journalist, daß der amerikanische Vertreter, Botschafter Christopher H. Phillips, in seinem politischen Hauptvortrag vor der Vollversammlung am 12. November 1970 hinsichtlich der chinesischen Repräsentationsfrage den Schwerpunkt nur auf die These gegen den Ausschluß Taipeis gelegt, aber keine einzige Kritik gegen Peking vorgebracht habe. Diese Stellungnahme habe - wie auch viele andere westliche Beobachter meinen - bei der Stimmenänderung zu Pekings Gunsten mitgewirkt ${ }^{5}$.

3 Vgl. die Leitartikel von Hsin-sheng Pao und Chung-kuo Shih-pao, 13. Nov. 1969.

4 Vgl. Chung-yang Jih-pao (Central Daily News), 21.-22. Nov. 1970.

5 Vgl. The Japan Times, 22. Nov. 1970. 
Am tiefsten muß die Regierung in Taipei wegen der Sinneswandlung einiger afrikanischer Staaten beunruhigt sein, da allein dieser Kontinent ungefähr zwei Fünftel der gesamten UNO-Stimmen besitzt und die meisten neuen Länder dieses Erdteils unter dem Motto „non-alignement“ außenpolitisch häufig zwischen dem Osten und dem Westen lavieren. Der Wettbewerb um die diplomatische Anerkennung zwischen Peking und Taipei ist hier seit eh und je krasser als anderswo ${ }^{6}$. Bis Anfang 1964 hatte Taipei bei dem Kopf-an-Kopf-Rennen diplomatische Beziehungen zu 18 unter den seinerzeit insgesamt 35 afrikanischen souveränen Staaten aufgenommen; Peking zu 15. Doch 1964 und 1965 geriet Taipeis Diplomatie in Afrika in eine schwere Krise. Auf die Anerkennung Pekings durch Frankreich (27. 1. 1964) zogen die folgenden afrikanischen Mitgliedstaaten der Französischen Gemeinschaft nach: Kongo-Brazzaville (18. 2. 1964), die Zentralafrikanische Republik (5. 11. 1964), Senegal (8. 11. 1964), Dahomey (8. 4. 1965) und Mauretanien (19. 7. 1965). Dies hatte, wie bereits erwähnt, zur Folge, daß 1965 die Stimmen für Peking und die für Taipei in der UNO zum ersten Mal ins Gleichgewicht gebracht wurden (47 gegen 47). Trotzdem konnte Taipei wegen der Verschlechterung der Beziehungen Pekings zu einigen afrikanischen Staaten erneut Beziehungen zu Dahomey (21. 4. 1966), zur Zentralafrikanischen Republik (6. Mai 1968) sowie Senegal (16. 7. 1969) anknüpfen. Dagegen verbesserte Peking mit der Anerkennung durch Aqquatorial-Guinea (15. 10. 1970), Äthiopien (2. 12. 1970) und Nigeria (10. 2. 1971) wieder seine Position. Zur Zeit unterhält Taipei diplomatische Beziehungen zu 22 afrikanischen Staaten - Botswana, Dahomey, Elfenbeinküste, Gabun, Gambia, Kamerun, Kongo (Kin.), Lesotho, Liberia, Libyen, Madagaskar, Malawi, Niger, OberVolta, Ruanda, Senegal, Sierra Leone, Südafrika (konsularische Beziehungen), Swaziland, Tschad, Togo und der Zentralafrikanischen Republik - Peking dagegen nur zu 16 - Algerien, Äquatorial-Guinea, Äthiopien, Guinea, Kenya, Kongo (Br.), Mali, Marokko, Mauretanien, Nigeria, Sambia, Somalia, Sudan, Tansania, Uganda und der VAR. Noch 5 der insgesamt 43 afrikanischen Staaten haben gegenwärtig weder Beziehungen zu Peking noch zu Taipei, nämlich Burundi (Unterbrechung der Beziehungen zu Peking im Januar 1965), Ghana (Unterbrechung der Beziehungen zu Peking im November 1966), Mauritius, Rhodesien (kein UNO-Mitglied) und Tunesien (Unterbrechung der Beziehungen zu Peking im September 1967. Nach einer Meldung von "Le Monde“ vom 8. 12. 1970 faßt Tunesien die Wiederaufnahme diplomatischer Beziehungen zur Volksrepublik China ins Auge).

Während Peking mit Kapitalhilfe und Ideologie seinen Einfluß in Afrika auszudehnen versucht, beschränkt sich Taipei bei der Verbesserung der Beziehungen zu den afrikanischen Staaten bewußt auf technische Hilfeleistungen, besonders im Bereich der Landwirtschaft ${ }^{7}$. Um sich die afrikanischen Stimmen in der UNO zu sichern, schickte Taipei darüber hinaus in den letzten Jahren jedes Mal vor Einberufung der Vollversammlung seinen Afrika-Experten, den Stellvertretenden Außenminister H. K. Yang, als Sonderbotschafter auf eine Marathon-Goodwill-Reise von dreimonatiger Dauer durch mehr als zwanzig afrikanische Staaten. Die unermüdlichen Bemühungen der nationalchinesischen Regierung haben zwar bislang gewisse Erfolge

6 Vgl. dazu Volker Matthies: China und Afrika, Mitteilungen des Instituts für Asienkunde Hamburg, Nr. 26, Hamburg 1969, S. 62 ff.; L. M. S. Slawecki: "The Two Chinas in Africa ${ }^{\circ}$, in: Foreign Affairs, Vol. 41, No. 2 (Jan. 1963), S. 398-409; G. T. Yu: "Chinese Rivalry in Africa“, in: Race, Vol. 5, No. 4 (April 1964), S. 35-47; ders.: "Peking versus Taipei in the World Arena: Chinese Competition in Africa ${ }^{\circ}$, in: Asian Survey, Vol. 3, No. 9 (Sept. 1963), S. 439-453; Africa and the Two Chinas: A Summary of Known Facts, in: Africa Report, Jan. 1965.

7 Mitte 1969 hatte Taipei insgesamt 25 Expertengruppen und 690 Experten in Afrika, dazu s. China Yearbook 1969-1970, S. $376 \mathrm{ff}$. 
gezeitigt, doch werden die afrikanischen Stimmen für Taipei in der UNO seit zwei Jahren immer unsicherer. Mauritius z. B., das erst 1968 von Großbritannien unabhängig wurde und sich durch mehrere gegenseitige Freundschaftsbesuche der Prominenten sowie durch Unterzeichnung einer bilateralen Vereinbarung über landwirtschaftlich-technische Zusammenarbeit vom Juli 1969 an Taipei annäherte, hat in demselben Jahr zur Überraschung der nationalchinesischen Regierung seine Stimme in der UNO für die Aufnahme Pekings abgegeben. 1.970 hat der Inselstaat jedoch abermals die Fronten gewechselt und bei der Abstimmung über Albaniens Antrag wieder für Taipei (nämlich gegen Pekings Aufnahme) gestimmt. Dagegen haben sich Botswana, Kamerun, Senegal und die Zentralafrikanische Republik ${ }^{7 a}$, die alle mit Taipei volle diplomatische Beziehungen unterhalten und 1969 für Taipei und gegen Peking gestimmt hatte, 1970 - auf den albanischen Antrag hin der Stimme enthalten. Außerdem haben Kamerun, Mauritius sowie Tschad über den amerikanischen Antrag (in bezug auf die "wichtige Frage“) Nein-Stimmen abgegeben. Aquatorial-Guinea gab 1970 seinen früheren neutralen Standpunkt auf und stimmte sowohl über den albanischen als auch den amerikanischen Antrag zugunsten Pekings. Libyen, wo die meisten nationalchinesischen Ärzte, Krankenschwestern und Ingenieure in Afrika arbeiten ${ }^{8}$ hat sich seit dem politischen Umsturz vom 1. September 1969 bei der UNO-Abstimmung Peking zugewendet, obwohl seine Beziehungen zu Taipei noch nicht unterbrochen sind.

Wenn man die afrikanischen Stimmen als Eckpfeiler der Position Taipeis in der UNO ansehen kann, so gilt die Unterstützung der lateinamerikanischen Staaten für Taipei als der eigentliche Eckstein. Mit 24 Mitgliedern ist Lateinamerika neben Afrika der zweitgrößte Staatenblock in der UNO. Bis 1969 hatte lediglich Kuba in der Vollversammlung die Aufnahme Pekings und gleichzeitig den Ausschluß Taipeis befürwortet. Doch Chile folgte 1970 dem Beispiel Kubas und gab die zweite lateinamerikanische Stimme für Peking ab. Unter der Regierung des Marxisten Allende erfolgte bald die diplomatische Anerkennung zwischen Santiago und Peking. Ecuador, Guayana, Jamaica und Trinidad-Tobago haben sich 1970 wie 1969 bei dem Antrag für die Aufnahme Pekings und den Ausschluß Taipeis der Stimme enthalten. Ihnen schlossen sich Bolivien und Peru 1970 zum ersten Mal an. Allerdings sind bei dem Antrag über die "wichtige Frage“ nur Guayana und TrinidadTobago neutral geblieben, während Bolivien, Ecuador und Jamaica dafür und Peru wie Chile mit Kuba dagegen gestimmt haben. Interessant ist auch die Stellungnahme von Barbados: es stimmte 1970 wie 1969 gegen Pekings Aufnahme und Taipeis Ausschluß, doch schwieg es sich über die von den USA aufgerichtete Sperre einer Zweidrittelmehrheit gegen Peking aus. Die schwankenden Tendenzen der lateinamerikanischen Stimmen machen der nationalchinesischen Regierung ähnliche Sorge wie die Entwicklung in Afrika.

In der Heimatregion Asien und im pazifischen Raum kann Taipei mit den ständigen Stimmen von Australien, Japan, den Philippinen, Thailand sowie Neuseeland rechnen. Peking genießt die zuverlässige Unterstützung von Afghanistan, Birma, Ceylon, Indien, der Mongolei, Nepal und Pakistan. Kambodscha, das die einstigen engen Beziehungen zur Volksrepublik China wegen Pekings Anerkennung der Exilregierung des gestürzten Prinzen Sihanouk unterbrochen und vor kurzem mit Nationalchina offizielle Handelsbeziehungen aufgenommen hat, stimmte 1970 in

\footnotetext{
7a Der Staatspräsident der Zentralafrikanischen Republik Jean Bedel Bokassa hatte noch im Oktober 1970, einen Monat vor der letzten UNO-Abstimmung, einen Staatsbesuch in Taiwan abgestattet.

8 China Yearbook 1969-1970, S. 377.
} 
der UNO bei der Abstimmung über die beiden Anträge zur Chinafrage erstmals gegen Peking und Taipei. Indonesien, das ebenfalls 1967 infolge des Militärputsches seine sämtlichen diplomatischen Vertreter aus der Volksrepublik China abberufen hatte, zeigt jedoch seine Zurückhaltung seit 1968 nur durch Nichtbeteiligung an der Abstimmung über alle Anträge zur Chinafrage. Ahnlich verhielt sich der Inselstaat Malediven im Jahre 1970: Er war bei der Abstimmung abwesend, obwohl er diplomatische Beziehungen zu Taipei unterhält und 1969 den Antrag der „wichtigen Frage“ noch unterstützt hatte. Die vor kurzer Zeit unabhängig gewordenen Fidschi-Inseln sowie das Königreich Laos, das nur formelle diplomatische Beziehungen zu Peking unterhält, enthielten sich der Stimme über den Antrag zur Aufnahme Pekings und zum Ausschluß Taipeis, stimmten jedoch für die „wichtige Frage“. Dagegen verneinten Malaysia und Singapur den Prozedurantrag der „wichtigen Frage", vermieden allerdings ebenfalls eine Stellungnahme zum Substanzproblem über den Repräsentationswechsel zwischen Peking und Taipei durch Stimmenthaltung. Taipei hat sich in den letzten Jahren darum bemüht, seine Beziehungen $z u$ den beiden Staaten zu verbessern, und hat dabei den Erfolg erzielt, daß in Kuala Lumpur ein Generalkonsulat und in Singapore eine offizielle Handelsmission eröffnet wurden. Malaysia hatte früher in der UNO immer gegen Pekings Aufnahme gestimmt. In Taipei führt man die veränderte Haltung Malaysias in der UNO auf seine neuen Bemühungen um eine Neutralisierung Südostasiens durch Peking, Moskau und Washington zurück ${ }^{9}$.

Ferner scheint auch die japanische Chinapolitik in Bewegung geraten zu sein, zumal seit Kanada und Italien Peking anerkannt haben. Angesichts des neuen Abstimmungsresultats in der UNO hat der japanische Außenminister im Parlament zum ersten Mal offen darauf hingewiesen, daß Japan in Zukunft nicht unbedingt die Frage der chinesischen Repräsentation als "wichtige Frage" betrachten werde ${ }^{10}$. Dennoch hat er dazu keine neuen politischen Richtlinien erkennen lassen. Die doppelzüngigen Äußerungen der japanischen Regierung zur Chinafrage sind nicht ganz neu. Aufgrund der japanischen Bindungen an Washington und Taipei ist ein vorschneller und dramatischer Wechsel der Chinapolitik Tokyos zumindest unter der jetzigen Regierung nicht sehr wahrscheinlich.

Im Nahen Osten ist das Verhältnis zwischen den Stimmen für Peking und den Stimmen für Taipei auch ungefähr gleich. All die Länder, die diplomatische Beziehungen zu Peking unterhalten, haben wie in früheren Jahren über die beiden Anträge bezüglich der Chinafrage für Peking gestimmt (Irak, Süd-Jemen, Syrien und Jemen). Andererseits haben auch Israel, Jordanien, Saudi-Arabien und die Türkei ihre Stellungnahme gegen Pekings Aufnahme und für die „wichtige Frage* 1970 nicht geändert. Unter ihnen hat nur Israel keine diplomatischen Beziehungen zu Taipei; andererseits hatte es zwar bereits 1950 Peking anerkannt ${ }^{11}$, aber bis jetzt sind noch keine diplomatischen Beziehungen zwischen den beiden Seiten zustande gekommen. Der Iran, Kuwait und der Libanon haben zwar diplomatische Beziehungen mit Taipei, wollten sich jedoch nicht über den Substanzantrag zur Aufnahme Pekings 1970 äußern, wie sie dies auch früher nicht getan hatten. Dennoch stimmten der Iran sowie der Libanon unverändert für den Prozedurantrag der „wichtigen Frage“, während Kuwait 1970 auf seine Enthaltung verzichtete und seine

9 Dazu vgl. Chung-yang Jih-pao, 22. Nov. 1970; Malaysia Digest, Vol. 2, No. 19, 16. Okt. 1970.

10 The Japan Times, 4. Dez. 1970.

11 Nach Jen-min Shou-ts'e (Volkshandbuch) 1965, Ta-kung-pao-she, S. 367. 
erste Nein-Stimme gegen die Auffassung abgab, daß eine Zweidrittelmehrheit für Pekings Aufnahme in die UNO erforderlich sei.

Größte Sorgen hat Taipeis Diplomatie in Europa. Nachdem Frankreich (1964) und Italien (1970) Peking anerkannt haben, bleiben nun nur noch Belgien, Griechenland, Luxemburg, Malta, Portugal, Spanien, Zypern und der Vatikan in Beziehungen zu Taipei. Abgesehen vom Vatikan, der nicht zur UNO gehört, lehnten darüber hinaus 1970 unter diesen Staaten lediglich noch Griechenland, Malta und Spanien bei den Abstimmungen der beiden Anträge die Aufnahme Pekings deutlich ab. Irland (keine Beziehungen mit Taipei und Peking) und Luxemburg, die vor 1969 Taipei noch voll unterstützt hatten, folgten 1970 dem Beispiel Belgiens, das seit 1969 nur den Prozedurantrag der "wichtigen Frage“ bejaht und $\mathrm{zu}$ dem Antrag auf Pekings Aufnahme durch Stimmenthaltung Stellung nahm. Die gleiche Haltung zeigten außerdem die Niederlande (diplomatische Beziehungen mit Peking), Island (weder Beziehungen zu Peking noch zu Taipei) und Zypern seit einigen Jahren. Portugal, das zwar, wie gesagt, diplomatische Beziehungen mit Taipei unterhält, aber wegen Macao ständig politische Angst vor Peking hat, bewahrte sich von jeher seine Neutralität bei den Abstimmungen aller Anträge zur Chinafrage in der UNO. Neu ist der Standpunkt Osterreichs und Italiens. Beide haben nach dem Vorbild von Großbritannien sowohl den Antrag für Pekings Aufnahme als auch für die "wichtige Frage“ mit ihren Stimmen unterstützt. Insgesamt sind unter den 27 europäischen Staaten 18 für und zwei gegen Pekings Aufnahme, bei 7 Enthaltungen, sowie 11 für und 15 gegen das Erfordernis einer Zweidrittelmehrheit für Pekings Aufnahme, bei 1 Enthaltung.

Nicht weniger als in Europa muß sich Taipei auch um die neue Entwicklung in Nordamerika kümmern. Kanada hat bereits Taipei den Rücken gekehrt. Wie Rom hat Ottawa nach seiner Anerkennung Pekings 1970 auch dessen Aufnahme in die UNO befürwortet, obwohl es um einer gewissen Solidarität mit den USA willen immer noch für eine Zweidrittelmehrheit eintrat. Selbst gegenüber Washington wird Taipei immer skeptischer. Längst hat die Presse in Taiwan Nixons Außenpolitik („Verhandlung statt Konfrontation“) gegenüber dem Ostblock kritisiert. Voller Mißtrauen steht Taipei insbesondere den von Nixon am 25. Juli 1969 auf der Pazifikinsel Guam proklamierten Prinzipien einer neuen Asienpolitik gegenüber, die vor allem die Verminderung der amerikanischen Intervention und der amerikanischen Truppen in Asien sowie Bemühungen um eine Verständigung mit Peking bedeuten ${ }^{12}$. Ferner läßt die neue Form von Washingtons Chinapolitik in der UNO - nicht mehr gegen Pekings Eintritt, sondern nur gegen Taipeis Ausschluß - Taipei befürchten, das „Weiße Haus“ könne den Weg einer „ZweiChina-Lösung " einschlagen ${ }^{13}$ und dadurch den Anspruch der Nationalregierung auf die Alleinvertretung Chinas weiter erschüttern.

\footnotetext{
12 Dazu s. Ch'en Shao-hsien: „Lun Mei-kuo-ti Hsin-ya-chou-cheng-ch'e ${ }^{\alpha}$ (Kommentar zu der neuen Asienpolitik der Vereinigten Staaten), in: Wen-t'i yü Yen-chu (Issues \& Studies), Vol. 9, No. 1 (Okt. 1969), S. $15 \mathrm{ff}$; vgl. auch die Leitartikel von Chung-kuo Shih-pao, 28. Juli 1969, und Chunghua Jih-pao, 1. Aug. 1969.

13 Dazu vgl. die Leitartikel von Chung-hua Jih-pao, 21. Nov. 1970, u. Kao-hsiung Hsin-wen-pao 26. Nov. 1970.
} 
Zwischen 1966 und 1968 wurden die Versuche Kanadas und Italiens für eine Doppelrepräsentation in der UNO bereits torpediert ${ }^{14}$. Taipei und Peking waren in dieser Frage einer Meinung, indem sie - wenngleich aus verschiedenen Gründen — den Gedanken einer „Zwei-China“-Theorie zurückwiesen. Angesichts der neuen Entwicklung ist keine neue politische Richtlinie Taipeis abzusehen. Sicherlich wird die nationalchinesische Regierung weiterhin auf ihrem Anspruch der Alleinvertretung Chinas bestehen. Um einen weiteren Stimmenverlust in der UNO zu vermeiden, sollen die bisherigen vorbeugenden Maßnahmen, nämlich die landwirtschaftliche technische Hilfe für afrikanische und lateinamerikanische Länder, in Zukunft noch weiter intensiviert werden. Dazu hat der nationalchinesische Stellvertretende Außenminister H. K. Yang gleich nach der UNO-Abstimmung über die Chinafrage am 1. Dezember 1970 im State Department über eine Kooperation mit den USA gesprochen ${ }^{15}$. Ferner wird eine Verstärkung von Taiwans Außenhandel mit den europäischen Ländern, zumal mit Belgien, erwartet ${ }^{16}$, da Brüssel wie Luxemburg und Wien nun auch eine Anerkennung Pekings in Erwägung zieht. Gegenüber den USA wird die Regierung in Taipei aufgefordert, die Informationsarbeit noch stärker zu fördern, um damit das Weitergehen von Washingtons „Beschwichtigungspolitik“ zu verhindern ${ }^{16 a}$. Letzten Endes meint man in Taiwan, daß die Repräsentationsfrage in der UNO nur durch „Rückeroberung des Festlands" für immer beseitigt werden könne'17. Außerdem glaubt die Regierung in Taipei, daß die UNO sich selbst aufgäbe, wenn sie Peking den Zutritt gestattete ${ }^{18}$.

14 Während der kanadische Plan für eine Zwischenlösung der Chinafrage durch eine Repräsentation der Republik China in der Vollversammlung und eine Repräsentation der Volksrepublik China in der Vollversammlung sowie im Sicherheitsrat als ständiger Mitgliedstaat mangels ausreichender Unterstützung 1966 überhaupt nicht auf die Tagesordnung der Vollversammlung gesetzt werden konnte, wurde die italienische Initiative für die Bildung eines Untersuchungskomitees zur Lösung der chinesischen Repräsentationsfrage dreimal - 1966 mit 34 . Ja- und 62 Nein-Stimmen bei 25 Stimmenthaltungen; $1967 \mathrm{mit} 32 \mathrm{Ja}$ - und 57 Nein-Stimmen bei 33 Stimmenthaltungen und 1968 mit $30 \mathrm{Ja}-$ und $67 \mathrm{Nein}-$ Stimmen bei 29 Stimmenthaltungen - von der Vollversammlung abgelehnt.

15 Meldung der Chung-yang Jih-pao, 3. Dez. 1970.

16 Vgl. Chung-vang Jih-pao, 2. Dez. 1970, Meldung über die Einberufung einer Konsulationskonferenz der wirtschaftlichen. Vertreter in Europa und Afrika; ebenda, 4. Dez. 1970, Meldung über die Gründung der chinesisch-belgischen Kommission für wirtschaftliche Zusammenarbeit.

16a Chung-yang Jih-pao, 15. Dez. 1970.

17 Vgl. die Leitartikel von Chung-yang Jih-pao, 21. Nov. 1970.

$18 \mathrm{Vgl}$. den Vortrag des nationalchinesischen Außenministers vor der UNO-Vollversammlung vom 12. Nov. 1970, Chung-yang Jih-pao, 20.-21. Dez. 1970; Erklärung des Sprechers des Außenministeriums auf einer Pressekonferenz in Taipei, ebenda, 28. Nov. 1970. 
Überblick des Stimmenverhältnisses 1970 über die Aufnahme Pekings

1 = Diplomatische Beziehungen mit P (Peking), $\mathrm{T}$ (Taipei) oder K (Keiner)

2 = Stellungnahme zur ,wichtigen Frage“: J (Ja)-, N (Nein)-Stimme oder E (Enthaltung) 1970

3 = Stellungnahme zur Aufnahme Pekings 1969

\begin{tabular}{|c|c|c|c|c|c|c|c|c|c|c|c|}
\hline \multicolumn{4}{|c|}{ Ja-Stimmen } & \multicolumn{4}{|c|}{ Nein-Stimmen } & \multicolumn{4}{|c|}{ Stimmenthaltung } \\
\hline Staat & 1 & 2 & 3 & Staat & 1 & 2 & 3 & Staat & 1 & 2 & 3 \\
\hline Afghanistan & $\mathrm{P}$ & $\mathrm{N}$ & $\mathrm{J}$ & Argentinien & $\mathrm{T}$ & $\mathrm{J}$ & $\mathrm{N}$ & Belgien & $\mathrm{T}$ & $\mathrm{J}$ & $\mathrm{E}$ \\
\hline Albanien & $\mathrm{P}$ & $\mathrm{N}$ & $\mathrm{J}$ & Australien & $\mathrm{T}$ & $\mathrm{J}$ & $\mathrm{N}$ & Bolivien & $\mathrm{T}$ & $\mathrm{J}$ & $\mathbf{N}$ \\
\hline Algerien & $\mathrm{P}$ & $\mathrm{N}$ & $\mathrm{J}$ & Barbados & $\mathrm{T}$ & $\mathrm{E}$ & $\mathrm{N}$ & Botswana & $\mathrm{T}$ & $\mathrm{J}$ & $\mathbf{N}$ \\
\hline Äquat.-Guinea & $\mathrm{P}$ & $\mathrm{N}$ & $\mathrm{E}$ & Brasilien & $\mathrm{T}$ & $\mathrm{J}$ & $\mathrm{N}$ & Ecuador & $\mathrm{T}$ & $\mathrm{J}$ & $\mathrm{E}$ \\
\hline Äthiopien & $\mathrm{Pa})$ & $\mathrm{N}$ & $\mathrm{J}$ & China (Rep.) & & $\mathrm{J}$ & $\mathrm{N}$ & Fidschi & $\mathrm{K}$ & $\mathrm{J}$ & \\
\hline Birma & $\mathrm{P}$ & $\mathrm{N}$ & $\mathrm{J}$ & Costa Rica & $\mathrm{T}$ & $\mathrm{J}$ & $\mathrm{N}$ & Guayana & $\mathrm{T}$ & $\mathrm{E}$ & $\mathrm{E}$ \\
\hline Bulgarien & $\mathrm{P}$ & $\mathrm{N}$ & $\mathrm{J}$ & Dahomey & $\mathrm{T}$ & $\mathrm{J}$ & $\mathrm{N}$ & Iran & $\mathrm{T}$ & $\mathrm{J}$ & $\mathrm{E}$ \\
\hline Burundi & $\mathrm{P}$ & $\mathrm{N}$ & $\mathrm{J}$ & Dominik. Rep. & $\mathrm{T}$ & $\mathrm{J}$ & $\mathrm{N}$ & Irland & $\mathrm{K}$ & $\mathrm{J}$ & $\mathbf{N}$ \\
\hline Ceylon & $\mathrm{P}$ & $\mathrm{N}$ & $\mathrm{J}$ & El Salvador & $\mathrm{T}$ & $\mathrm{J}$ & $\mathrm{N}$ & Island & $\mathrm{K}$ & $\mathrm{J}$ & $\mathrm{E}$ \\
\hline Chile & $\mathrm{Pa})$ & $\mathrm{N}$ & $\mathrm{E}$ & Elfenbeinküste & $\mathrm{T}$ & $\mathrm{J}$ & $\mathrm{E}$ & Jamaica & $\mathrm{T}$ & $\mathrm{J}$ & $\mathrm{E}$ \\
\hline Dänemark & $\mathrm{P}$ & $\mathrm{N}$ & $\mathrm{J}$ & Gabun & $\mathrm{T}$ & $\mathrm{J}$ & $\mathbf{N}$ & Kamerun & $\mathrm{T}$ & $\mathrm{E}$ & $\mathbf{N}$ \\
\hline Finnland & $\mathrm{P}$ & $\mathrm{N}$ & $\mathrm{J}$ & Gambia & $\mathrm{T}$ & $\mathrm{J}$ & $\mathbf{N}$ & Kuwait & $\mathrm{T}$ & $\mathbf{N}$ & $\mathrm{E}$ \\
\hline Frankreich & $\mathrm{P}$ & $\mathrm{N}$ & $\mathrm{J}$ & Griechenland & $\mathrm{T}$ & $\mathrm{J}$ & $\mathrm{N}$ & Laos & $\mathrm{P}$ & $\mathrm{J}$ & $\mathrm{E}$ \\
\hline Ghana & $\mathrm{K}$ & $\mathrm{N}$ & $\mathrm{J}$ & Guatemala & $\mathrm{T}$ & $\mathrm{J}$ & $\mathrm{N}$ & Libanon & $\mathrm{T}$ & $\mathrm{J}$ & $\mathrm{E}$ \\
\hline Großbritannien & $\mathrm{P}$ & $\mathrm{J}$ & $\mathrm{J}$ & Haiti & $\mathrm{T}$ & $\mathrm{J}$ & $\mathrm{N}$ & Luxemburg & $\mathrm{T}$ & $\mathrm{J}$ & $\mathbf{N}$ \\
\hline Guinea & $\mathrm{P}$ & $\mathrm{N}$ & $\mathrm{J}$ & Honduras & $\mathrm{T}$ & $\mathrm{J}$ & $\mathrm{N}$ & Malaysia & $\mathrm{K}^{\mathrm{b})}$ & $\mathrm{N}$ & $\mathbf{N}$ \\
\hline Indien & $\mathrm{P}$ & $\mathrm{N}$ & $\mathrm{J}$ & Israel & $\mathrm{K}$ & $\mathrm{J}$ & $\mathrm{N}$ & Niederlande & $\mathrm{P}$ & $\mathrm{J}$ & $\mathrm{E}$ \\
\hline Irak & $\mathrm{P}$ & $\mathrm{N}$ & $\mathrm{J}$ & Japan & $\mathrm{T}$ & $\mathrm{J}$ & $\mathrm{N}$ & Peru & $\mathrm{T}$ & $\mathrm{N}$ & $\mathrm{N}$ \\
\hline Italien & $\mathrm{P}$ & $\mathrm{J}$ & $\mathrm{E}$ & Jordanien & $\mathrm{T}$ & $\mathrm{J}$ & $\mathrm{N}$ & Portugal & $\mathrm{T}$ & $\mathrm{E}$ & $\mathrm{E}$ \\
\hline Jemen & $\mathrm{P}$ & $\mathrm{N}$ & $\mathrm{J}$ & Kambodscha & $\mathrm{K}$ & $\mathrm{J}$ & $\mathrm{J}$ & Senegal & $\mathrm{T}$ & $\mathrm{J}$ & $\mathrm{N}$ \\
\hline Jugoslawien & $\mathrm{P}$ & $\mathrm{N}$ & $\mathrm{J}$ & Kolumbien & $\mathrm{T}$ & $\mathrm{J}$ & $\mathrm{N}$ & Singapur & $\mathrm{K}^{\mathrm{c})}$ & $\mathrm{N}$ & $\mathrm{E}$ \\
\hline Kanada & $\mathrm{P}$ & $\mathrm{J}$ & $\mathrm{E}$ & Kongo (Kin.) & $\mathrm{T}$ & $\mathrm{J}$ & $\mathrm{N}$ & Trinid Tobago & $\mathrm{K}$ & $\mathrm{E}$ & $\mathrm{E}$ \\
\hline Kenya & $\mathrm{P}$ & $\mathrm{N}$ & $\mathrm{J}$ & Lesotho & $\mathrm{T}$ & $\mathrm{J}$ & $\mathrm{N}$ & Tunesien & $\mathrm{K}$ & $\mathrm{N}$ & $\mathrm{E}$ \\
\hline Kongo (Br.) & $\mathrm{P}$ & $\mathrm{N}$ & $\mathrm{J}$ & Liberia & $\mathrm{T}$ & $\mathrm{J}$ & $\mathbf{N}$ & Zentr. Afr. Rep. & $\mathrm{T}$ & $\mathrm{J}$ & $\mathbf{N}$ \\
\hline Kuba & $\mathrm{P}$ & $\mathrm{N}$ & $\mathrm{J}$ & Madagaskar & $\mathrm{T}$ & $\mathrm{J}$ & $\mathrm{N}$ & Zypern & $\mathrm{T}$ & $\mathrm{J}$ & $\mathrm{E}$ \\
\hline Libyen & $\mathrm{T}$ & $\mathrm{N}$ & $\mathrm{J}$ & Malawi & $\mathrm{T}$ & $\mathrm{J}$ & $\mathrm{N}$ & Indonesien & & & \\
\hline Mali & $P$ & $\mathbf{N}$ & $\mathrm{J}$ & Malta & $\mathrm{T}$ & $\mathrm{J}$ & $\mathbf{N}$ & (Nichtbeteiligung & & & \\
\hline Marokko & $\mathrm{P}$ & $\mathrm{N}$ & $\mathrm{J}$ & Mauritius & $\mathrm{K}$ & $\mathrm{E}$ & $\mathrm{J}$ & 1969 und 1970) & & & \\
\hline
\end{tabular}




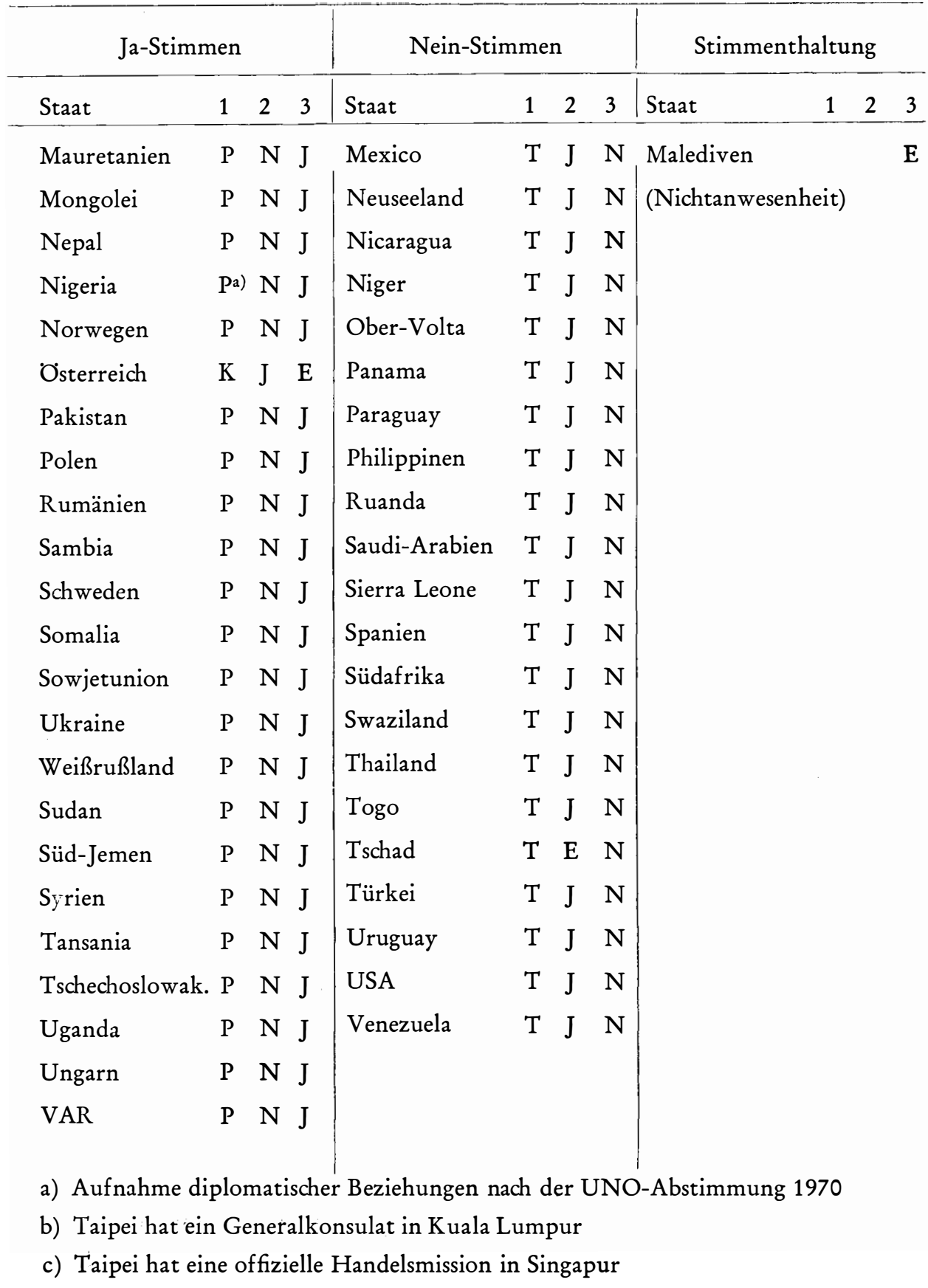

